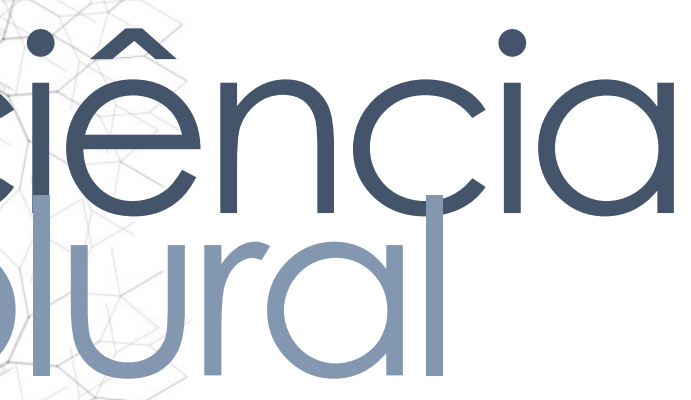

\title{
A EDUCAÇÃO POPULAR EM SAÚDE COM GRUPOS DE IDOSOS DIABÉTICOS NA ESTRATÉGIA SAÚDE DA FAMÍLIA: UMA PESQUISA-AÇÃO
}

Popular education in health with groups of diabetic elderly in the Family Health Strategy: an Action-Research

Marise Soares Almeida • Enfermeira da Secretaria Municipal de Saúde de Natal (RN);:• Mestre em Saúde da Família (RENASF/UFRN) • E-mail: marise-sa@hotmail.com

Ricardo Henrique Vieira de Melo • Dentista da Estratégia Saúde da Família de Nazaré (SMS Natal/RN) • Mestre em Saúde da Família (RENASF/UFRN) - Doutorando em Ciências da Saúde (Faculdade de Medicina do ABC - FMABC - Santo André/SP) • E-mail: ricardohvm@gmail.com

Rosana Lúcia Alves de Vilar • Docente do Departamento de Enfermagem (UFRN) • Doutora em Ciências Sociais (UFRN) • E-mail: rosanaalvesrn@gmail.com

Alexandre Bezerra Silva • Universidade Estadual do Rio Grande do Norte (UERN) • Enfermeiro Mestre em Saúde da Família (Renasf/UFRN) •

E-mail: alexandre_enfe@hotmail.com

Mércia Lima de Melo • Universidade Federal do Rio Grande do Norte • Acadêmica do Curso de Licenciatura em Educação Física (UFRN) • E-mail: mercialimamelo@gmail.com

Antônio Medeiros Júnior • Universidade Federal do Rio Grande do Norte • Doutor em Ciências da Saúde (UFRN) • Docente do Departamento de Saúde Coletiva (UFRN) • E-mail: soriedemjunior@gmail.com

Autor responsável pela correspondência

Ricardo Henrique Vieira de Melo• E-mail: ricardohvm@gmail.com 


\section{RESUMO}

Introdução: A Educação Popular em Saúde (EPS) remete indivíduos e grupos à troca de saberes e experiências, permitindo-lhes associar a saúde ao resultado das suas condições de vida levando a uma emancipação do sujeito. Objetivo: Promover ações educativas com portadores de Diabetes Mellitus (DM); realizar oficinas de capacitação em EPS com trabalhadores de saúde; e avaliar os resultados das ações realizadas para os trabalhadores e usuários, em uma Unidade de Saúde da Família em Natal/RN. Método: Trata-se de uma pesquisa-ação com o referencial teórico da Teoria da Educação Libertadora, centrada na pedagogia problematizadora. Participaram da pesquisa trinta trabalhadores de saúde e trinta e seis usuários diabéticos. As ações foram organizadas através de rodas de conversa, dinâmicas de grupo, narrativas de vida, relatos de experiências, e explicitação de saberes, desejos, limitações, crenças e valores socialmente construídos. A coleta dos dados foi realizada através da Técnica de Associação Livre de Palavras, entrevista semiestruturada e grupo focal. O material empírico foi submetido à análise de ocorrência com auxílio do programa IRAMUTEQ. Resultados: Foram geradas palavras, expressões e categorias, a partir dos temas abordados e de situações criativas mostrando que a EPS vem sendo incorporada timidamente no processo educativo dos sujeitos deste estudo e bem distante dos princípios de participação, organização de um trabalho político, ampliação dos espaços de diálogo, respeito, de solidariedade e tolerância entre os diversos atores envolvidos no enfrentamento dos problemas de saúde, fundamentais para o aperfeiçoamento na construção de práticas saudáveis da atenção básica. Conclusões: A utilização de práticas ativas de ensino-aprendizagem, centradas na ampliação da escuta e em capacitações sobre EPS, poderá possibilitar mudanças no cenário onde os usuários e trabalhadores de saúde atuam com a diabetes mellitus.

Palavras-Chave: Educação em saúde; Saúde da Família; Diabetes Mellitus. 


\section{ABSTRACT}

Introduction: Popular Health Education (EPS) refers to individuals and groups to exchange knowledge and experiences, allowing them to associate health to the outcomes of their living conditions. Objective: To know and promote educational actions with patients with diabetes Mellitus (DM); Perform training workshops in EPS with health workers; To know and develop participatory educative actions with diabetic groups; and evaluate the results of the actions performed for workers and users, in a family health unit in Natal/RN. Methods: This is an action research with the theoretical framework of liberating education theory, centered on problematizing pedagogy. Thirty health workers and 36 diabetic users participated in the study. The actions were organized through conversation wheels, group dynamics, life narratives, reports of experiences, and explication of knowledge, desires, limitations, beliefs and socially constructed values. Data collection was performed through the free word association technique, semistructured interview and focal group. The empirical material was subjected to occurrence analysis (Bardin) with the aid of the Iramuteq program (Ratinaud; Marchand). Results: The data analyses originated words, expressions, categories, themes and creative situations showing that EPS is in process of construction, but still very incipient in primary care. words, expressions and categories were generated from the themes addressed and creative situations showing that the EPS has been incorporated timidly in the educational process of the subjects of this study and far from the principles of participation, organization of a political work, expansion of the spaces of dialogue, respect, solidarity and tolerance among the various actors involved in coping with health problems, fundamental for the improvement in the construction of Healthy practices of primary care. Conclusions: The use of active teaching-learning practices, centered on the amplification of listening and in training on EPS, may enable changes in the scenario where users and health workers act with diabetes mellitus.

Keywords: Health Education, Family Health, Diabetes Mellitus. 


\section{Introdução}

No mundo e no Brasil é possível constatar um importante crescimento das condições humanas consideradas crônicas. Diversas mudanças observadas ao longo dos anos contribuíram para isso, tais como as demográficas, os novos padrões de consumo e estilos de vida, a urbanização, as estratégias do mercado econômico, dentre outras. Os índices de morbidade por Diabetes Mellitus (DM) têm tomado proporções de uma pandemia. Diante disso, e considerando que a população brasileira está envelhecendo, os profissionais de saúde identificaram a necessidade de assumir uma postura mais proativa que reativa no desenvolvimento de atividades educativas, buscando assim a melhoria da condição de saúde dos portadores dessa patologia ${ }^{1}$.

A prevenção do diabetes e de suas complicações é hoje prioridade de saúde pública, determinando a necessidade de adoção de novas estratégias para monitorar e enfrentar esta nova realidade. Na atenção básica, segundo Vasconcelos ${ }^{2}$, essa prevenção pode ser realizada por meio da vigilância de fatores de risco como sedentarismo, obesidade e hábitos alimentares não saudáveis; da identificação e tratamento de indivíduos de alto risco para diabetes (prevenção primária); da identificação de casos não diagnosticados de diabetes para tratamento (prevenção secundária); e da intensificação do controle de pacientes já diagnosticados visando prevenir complicações agudas e crônicas (prevenção terciária).

Existem diferenças significativas entre abordagens individuais e coletivas. Trabalhar coletivamente os aspectos educativos sobre as temáticas se mostrou mais efetivo. No desenvolvimento das atividades de educação em saúde, em rupos, é importante que os profissionais enfatizem, também, o empoderamento autonomia do sujeito para reforçar o desenvolvimento de habilidades e motivar ra um cuidado mais integral ${ }^{3,4}$. 
Tendo em vista a complexidade de situações enfrentadas pelas equipes de saúde da família, os trabalhos com grupos não deveriam ser considerados ações substitutivas e compensatórias dos atendimentos individuais. A postura dos trabalhadores de saúde em transferir conhecimentos, sem motivar a população, necessita de estratégias que abordem o cuidado através do acolhimento, vínculo e responsabilização utilizando dinâmicas mais integrativas ${ }^{5}$.

Nas práticas de saúde, a simples transferência de informações nem sempre surte o efeito desejado. Nesse sentido, é importante que a educação da pessoa com diabetes seja priorizada em espaços que permitam o diálogo, onde serão debatidos problemas específicos conectados às condições de vida e à dinâmica comunitária, de tal forma que os cidadãos envolvidos sejam valorizados na sua explicitação de saberes, incorporações e reflexões. Só então a incorporação de conhecimentos e práticas será possível entre os cidadãos, suas famílias e os grupos ${ }^{6}$.

Dessa forma, o estudo sobre grupos de diabéticos torna-se relevante por tratar-se de um problema atual, presente na realidade de saúde pública brasileira no âmbito da atenção primária. Sabe-se que o conhecimento sobre DM, sedimentado a partir do intercambio com vários atores sociais, deixará o paciente mais crítico sobre seu estado de saúde e as múltiplas dimensões da doença.

A complicação mais séria da DM é a gangrena nos pés, resultado de úlceras não cuidadas. Cerca de $5 \%$ dos indivíduos com diabetes eventualmente são submetidos à amputação de um pé. A prevalência de amputações em decorrência do pé diabético tem um índice elevado, principalmente em artelhos. É uma complicação que se apresenta e uma grande variação na faixa etária, de 23 a 80 anos, por isso a importância de estratégias adequadas para o tratamento reventivo em cada contexto ${ }^{7}$.

Apesar da ampliação da rede de assistência básica, prestada através das uipes de saúde da família, observa-se uma grande frequência de pacientes 
portadores de úlceras nos pés, hospitalizados, rumando para cirurgias mutiladoras. Cerca da metade dos pacientes diabéticos com amputações da extremidade inferior irá perder, eventualmente, outra perna em cinco anos. A gangrena de membros inferiores pode ser evitada em $90 \%$ dos casos, se houver controle adequado dos níveis de glicose no sangue e um cuidado diário com os pés. O DM tipo II, que corresponde a $90 \%$ dos casos de diabetes, é justamente o tipo de diabetes em que o indivíduo está mais propenso a desenvolver a úlcera diabética ${ }^{8}$.

A identificação precoce dos pacientes com alto risco de desenvolverem uma ulceração, portanto mais susceptível à amputação, é essencial para o sucesso de uma prevenção. O Consenso Internacional sobre o Pé Diabético, em 2001, criou um sistema de classificação de risco do pé diabético em três categorias e sugerindo a frequência da avaliação: Categoria 0, sem neuropatia; categoria 1, com neuropatia; categoria 2, com neuropatia e/ou deformidades e a categoria 3, amputação/ úlcera prévia 9 .

O cuidado integral com o paciente com diabetes e sua família tende a ser mais bem enfrentado por meio de práticas ativas e participativas, durante reuniões de grupo, levando em consideração aspectos culturais e psicossociais, com ênfase no empoderamento e na autonomia do paciente para seu autocuidado. Estimular os pacientes a se organizarem em grupos de ajuda mútua, como, por exemplo, grupo de caminhada, troca de receitas de alimentos diet, técnicas de autocuidado, entre outros ${ }^{10}$.

A Educação Popular em Saúde (EPS), em sua dimensão libertadora, remete indivíduos e grupos à troca de saberes e experiências, permitindo-lhes associar a aúde ao resultado das suas condições de vida levando a uma emancipação do ujeito. Sob essa ótica, trabalhadores e usuários da saúde são sujeitos do processo ucativo ${ }^{11,12}$. 
Uma grande contribuição para essa mudança de perspectiva em relação à saúde veio com Freire ${ }^{13}$. Ele tinha, por base, que toda aprendizagem humana, toda ação educativa deveria ser fundamentada no amor. É a pedagogia da condição humana, de sempre estar voltada para uma prática alegre e afetiva, porém embasada cientificamente e com domínio técnico a serviço da sociedade. Portanto, é importante que se deixem de lado as ações automáticas e impulsivas, para enxergar as pessoas e suas relações, não apenas suas doenças, fugindo da familiaridade e das rotinas.

Diante do contexto, surgiram diversas indagações, tipo: Como os profissionais de saúde podem contribuir para a transformação de um sujeito sem criatividade, em um sujeito autônomo? Quais as ações educativas utilizadas na orientação de usuários diabéticos? Quais metodologias participativas podem ser utilizadas? Como a EPS pode contribuir na construção e no apoio aos grupos sociais? Como transformar práticas pedagógicas tradicionais em práticas que levem o sujeito a superar situações que limitam o seu viver?

Esses questionamentos remetem à condição humana, com todas as suas peculiaridades, seus problemas individuais, coletivos e globais, e motivou à realização desta pesquisa. Sendo assim, os objetivos da investigação foram: Promover ações educativas com portadores de Diabetes Mellitus (DM); realizar oficinas de capacitação em EPS com trabalhadores de saúde; e avaliar os resultados das ações realizadas para os trabalhadores e usuários, em uma Unidade de Saúde da Família em Natal/RN. 


\section{Metodologia}

Trata-se de uma pesquisa-ação na qual foi utilizado, para a dinamização de três grupos educativos com reuniões periódicas mensais, o referencial teórico da Teoria da Educação Libertadora13, que tem como base uma pedagogia problematizadora e que valoriza o diálogo no processo de compreensão de si mesmo e do mundo.

Segundo Thiollent ${ }^{14}$, a pesquisa-ação assume um caráter emancipatório, onde os sujeitos da pesquisa passam a ter a oportunidade de se libertar de mitos e preconceitos, tomando consciência dia após dia das transformações que vão ocorrendo em si próprios.

O referencial Freireano desenvolve uma pedagogia fundamentada na realidade de vida e na consciência crítica dos educandos, buscando novas formas de aprendizado que não a repetição, a automaticidade, a prática de uma educação consciente, problematizadora, propondo a humanização das relações e a libertação dos homens ${ }^{15}$.

Participaram da pesquisa trinta trabalhadores de saúde e trinta e seis usuários diabéticos, moradores da área de abrangência da Unidade Saúde da Família (USF). Os critérios de inclusão dos usuários foram: ter diagnóstico de diabetes tipo 1 ou 2, morar nas áreas cadastradas pelas equipes, não possuir nenhum distúrbio cognitivo, aderir voluntariamente a pesquisa, desejar participar de reuniões mensais no salão da unidade e querer assinar o termo de consentimento livre e esclarecido. E os critérios de inclusão dos profissionais foram: fazer parte das equipes de saúde da unidade, desejar participar de forma oluntária da pesquisa e das reuniões mensais.

Cada grupo de usuários tinha, em média, doze participantes, e as ações oram organizadas no salão da unidade a partir do estímulo às relações 
horizontalizadas entre os sujeitos através de rodas de conversa, dinâmicas de grupo, narrativas de vida, relatos de experiências, exibições e discussões de filmes, músicas, explicitação de saberes, desejos, limitações, crenças e valores socialmente construídos.

A coleta dos dados foi realizada através da Técnica de Associação Livre de Palavras (TALP) e Rodas de Conversa. A TALP é uma técnica que atua sobre a estrutura psicológica do sujeito, tornando-a evidenciada a partir das quatro principais condições de um teste projetivo como: estimular, tornar observável, registrar e obter a comunicação verbal ${ }^{16}$. O material empírico foi organizado e submetido a duas análises: conteúdo temático e análise de ocorrência ${ }^{17}$; e análise pelo software IRAMUTEQ (Interface de R pour les Analyses Multidimensionnelles de Textes et de Questionnaires) ${ }^{18}$.

O IRAMUTEQ é uma ferramenta de informática gratuita e de fonte aberta, capaz de viabilizar diferentes tipos de análise de corpus textuais, desde a lexicografia básica (cálculo de frequência de palavras) até a análises multivariadas (análise de similitude e classificação hierárquica descendente) ${ }^{18}$.

A figura 1 ilustra a divisão do processo de pesquisa-ação em quatro fases, proposta por Thiollent ${ }^{14}$ : (1) fase exploratória, (2) fase principal, (3) fase de ação e (4) fase de avaliação. O objetivo dessa divisão foi facilitar a compreensão do processo e guiar a condução do trabalho. Como não são fases fixas e perfeitamente delimitadas, o caráter participativo e cooperativo, que enriquece a pesquisa-ação, não foi subestimado. 
Figura 1. Concepção e organização da pesquisa "A educação popular em saúde com grupos de idosos diabéticos na estratégia saúde da família". Natal-RN. 2018.

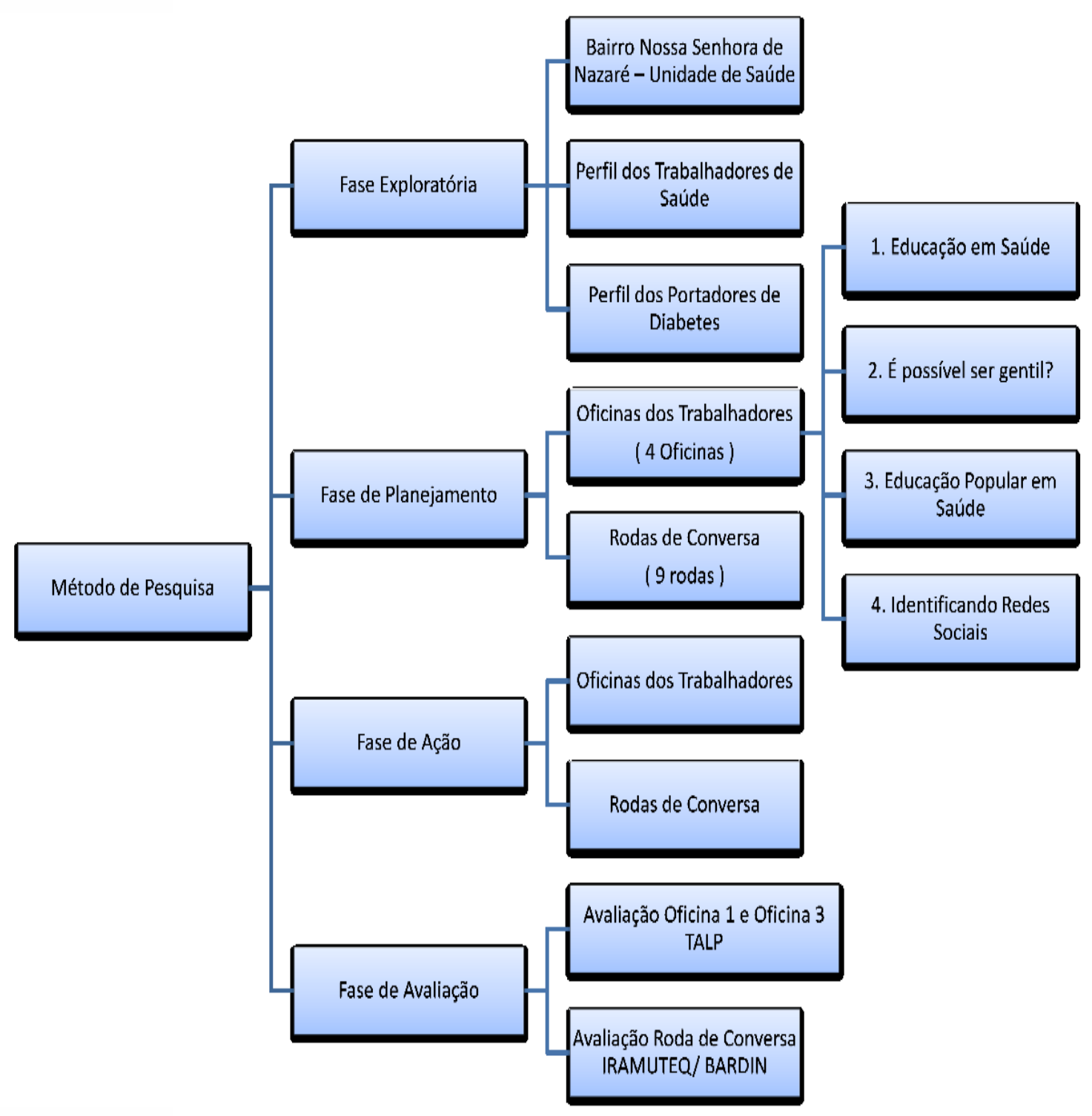

A investigação respeitou os protocolos éticos recomendados na Resolução 466/2012 do Conselho Nacional de Saúde que contempla as Diretrizes Regulamentadoras de Pesquisa envolvendo seres humanos, sendo submetido ao 
Comitê de Ética em Pesquisa (CEP) do Hospital Universitário Onofre Lopes (HUOL) via parecer $n^{\circ} 812.057 / 2014$.

\section{Resultados e Discussão}

\section{A fase exploratória}

A pesquisa foi realizada no município de Natal - $R N$, na região administrativa oeste, no bairro de Nossa Senhora de Nazaré, em uma Unidade de Saúde da Família. O bairro surgiu na década de 1950 e possui uma população de 42.795 habitantes distribuídos territorialmente em $864,341 \mathrm{Km}^{2}$, sendo que, menos de 50\% possui cobertura pela Estratégia Saúde da Família. A população residente é composta por $53,43 \%$ de mulheres e $46,57 \%$ de homens. O saneamento residencial alcança 92,33\% o acesso à água tratada é $90,89 \%$. O bairro possui $90 \%$ de drenagem e $95 \%$ de ruas pavimentadas. Existem duas praças, oito escolas, sendo quatro públicas e quatro privadas. Uma creche particular e uma pública. Tem um conselho comunitário e nenhum serviço de segurança pública. A Unidade de Saúde da Família possui 16 micro áreas, compondo três equipes de Saúde da Família e de Saúde Bucal19.

No Sistema de Informação da Atenção Básica (SISAB) existiam, na época do estudo, 240 usuários portadores de DM, sendo 73,09\% de sexo feminino, com ensino fundamental incompleto $(55,41 \%)$, com fatores de risco associados a diabetes em 86,7\% dos indivíduos, destacando-se o sedentarismo como o fator mais prevalente $(73,6 \%)$. A hipertensão arterial estava presente como patologia associada à DM em 79,3\% das pessoas.

O tempo de diagnóstico da diabetes estava entre cinco e nove anos, epresentando $45,0 \%$ dos portadores dessa condição. Uma curiosidade é que 2,5\%

diabéticos apresentavam maior renda e a maior escolaridade. Curioso, tretanto, é o fato de a segunda maior renda média ser destinada ao grupo de 
usuários diabéticos analfabetos. Cerca de 28,21\% eram de aposentados e 56,38\% de donas de casa (do lar) sem renda própria. Os antecedentes familiares com diabetes estavam presentes em $72,5 \%$ dos participantes, o que sugere um possível reflexo tanto de fatores genéticos quanto de maus hábitos herdados no coletivo ampliado da família.

Destaca-se que, nas características clínicas, entre os membros do grupo, $31,6 \%$ estavam classificados no primeiro grau de obesidade e desses, $26,3 \%$ eram sedentários. O fator de risco mais presente foi o sedentarismo, totalizando $71,1 \%$. A prevenção das complicações do diabetes se dá não só pelo controle da obesidade quanto pelos efeitos dos fitonutrientes presentes nas frutas e vegetais. A obesidade e a hiperlipidemia também apresentaram números consideráveis, com $30 \%$ e $27,5 \%$, respectivamente. O outro fator de risco bastante importante, o tabagismo, aparece em apenas $12,5 \%$ dos participantes. Ressalta-se a hipertensão arterial sistêmica como a patologia associada mais presente em $75 \%$ dos usuários diabéticos.

A vasculopatia e a alergia também estavam associadas, representando $47,5 \%$ e $32,5 \%$ respectivamente. As câimbras ou fraqueza muscular eram os sintomas mais presentes em $23,7 \%$ dos usuários. O aumento da renda não foi um fator determinante no uso de sapatos adequados. Nem todos os que tinham um nível mais elevado de escolaridade usavam sapatos adequados, entretanto, todos os que usavam sapatos adequados faziam parte da metade mais instruída do grupo. A autonomia e conscientização de cada sujeito é fator decisivo, independentemente do acesso à informação e da escolaridade, quanto ao uso do sapato adequado e ao cuidado com os pés.

Quanto ao exame do monofilamento, 15\% apresentaram sensibilidade Iterada. Desses, dois terços não utilizavam sapatos adequados. A presença de sibilidade alterada juntamente com o uso de sapatos inadequados pode trazer, 
em curto prazo, o aparecimento de úlceras que, quando não tratadas precocemente, podem ou não evoluir para amputação de membros inferiores.

Em relação ao tempo médio de atividade profissional dos trabalhadores de saúde, verifica-se que as médias apresentam um valor mínimo de 15 anos, mostrando que a equipe é bastante experiente. Por sua vez, o tempo médio de atividade profissional dedicado à ESF apresenta um valor mínimo de três anos, sendo que alguns profissionais como Enfermeiros, Técnicos de Enfermagem e Médicos possuem, em média, mais de oito anos dedicados à ESF. Quanto à composição das equipes, observamos que os Agentes Comunitários de Saúde e os Técnicos de Enfermagem são os mais numerosos, representando 34,5\% e 20,7\% do total dos demais membros, que são: Enfermeiros (13,8\%); Médicos (6,9\%); e somando 24,1\% - Auxiliar de Saúde Bucal, Odontólogo, Educador Físico e Assistente Social.

\section{As fases de planejamento e de ação}

Foram planejadas e realizadas quatro oficinas para os trabalhadores de saúde e seis Rodas de Conversa com os grupos de portadores de diabetes. As oficinas foram: 1) Educação em saúde; 2) É possível ser gentil; 3) Educação Popular em Saúde; e 4) Identificando as redes sociais de apoio aos diabéticos. Cada oficina foi formatada em três momentos: acolhimento e aplicação da técnica TALP; leitura de textos com apresentação das sínteses e confronto com as práticas de saúde locais; e problematização da realidade com propostas de ações transformadoras mediadas por momentos de reflexão crítica, dialógica e compartilhada.

E as rodas de conversa foram divididas didaticamente em cinco etapas para facilitar a compreensão dos participantes: acolhimento; escolha do tema; ontextualização; problematização; e finalização com rituais de agregação e onotação de aprendizagem. Nos momentos pós-rodas ainda estavam previstos omentos de escuta, acolhendo aquele participante que solicitasse ajuda para 
solucionar alguma necessidade particular. $\mathrm{O}$ áudio das falas dos participantes foi gravado para posterior transcrição e análise de conteúdo.

A Educação em Saúde, de forma mais participativa, surge como uma estratégia capaz de mostrar resultados positivos onde os profissionais têm a oportunidade de encontrar soluções coletivas para o enfretamento de questões reais vividas pela comunidade ${ }^{20}$.

Um estudo realizado por Torres; Pereira; Alexandre ${ }^{21}$ mostrou evidências de que a interação entre profissional e indivíduo, com base no diálogo, propicia mudanças de comportamento e melhoramento no autogerenciamento da doença. Além disso, identifica também a necessidade de os profissionais de saúde aprender mais sobre o processo e os métodos de educação em saúde, bem como a observação dos indivíduos e da comunidade. Ouvir os indivíduos e refletir conjuntamente sobre as vivências e percepções é fundamental para este processo. Apresentamos abaixo uma síntese dos resultados mais significativos durante o processo de realização das atividades planejadas.

\section{A fase de avaliação}

Os resultados da TALP sobre Educação em Saúde na Estratégia de Saúde da Família, apontaram a existência de conhecimentos sobre educação em saúde entre eles. Considerando se tratar de uma força de trabalho experiente, já que o tempo médio de profissão era de 15 anos, foram verificadas dificuldades de domínio de práticas pedagógicas mais participativas através de metodologias mais críticas, reflexivas, dialógicas e compartilhadas. As palavras mais repetidas e as mais importantes que cada um expôs sobre o tema foram: Prevenção (44\%); Trabalho (25\%); Coletividade (19\%); Saúde (19\%). E as palavras mais circuladas devido a sua importância para o trabalhador de saúde foram: Prevenção (19\%); e Equipe $(13 \%)$. 
As concepções sobre Educação em Saúde na Estratégia Saúde da Família revelaram compreensões diversas sobre a temática, compartilhando a vontade de educar, transmitir ensinamentos, mesmo sem considerar muito o conhecimento prévio da população e sua historicidade:

É compreendida como meio de levar e trazer conhecimentos na esfera dos objetivos da estratégia, assim como forma de discussão na busca de uma melhor atuação à saúde coletiva (Trabalhador 1).

Ação educativa com a comunidade com a finalidade de trocar conhecimentos relacionados à saúde. Feedback entre profissionais $e$ usuários da ESF (Trabalhador 3).

É um mecanismo ou uma forma de aprender, ensinar e compreender como se cuida da saúde (Trabalhador 7).

É um trabalho difícil que precisa ser desenvolvido em equipe porque se procura modificar toda a cultura e estilo de vida dos individuos. É promover conhecimento para os individuos para modificar os hábitos de vida para prevenir e promover a saúde (Trabalhador 10).

Com relação à Educação Popular em Saúde As palavras mais citadas foram: Acolhimento (43\%); Educação (29\%); Escuta (29\%); Orientação (29\%). E as palavras mais circuladas foram: Acolhimento (14\%); e Humanização (14\%). As concepções dos participantes sobre EPS na Estratégia Saúde da Família estão representadas nas falas a seguir:

Entendo que é o profissional atuante, que aproveitando a cultura do paciente o mesmo passa a orientar de acordo com a cultura dele (Trabalhador 1).

Educar com ações. Prevenções como: saúde da criança, adulto, adolescente etc. E a formação de grupos de gestantes, hipertensos, diabéticos, e os acompanhamentos (Trabalhador 3).

É tentar respeitar todas as crenças e somar junto com a educação formal a resolutividade das práticas de saúde juntamente com a comunidade (Trabalhador 6).

Forma de educar, de fazer compreender, levando em consideração os conhecimentos prévios dessa comunidade (Trabalhador 11). 


\section{ciência}

A análise do corpus "fala dos trabalhadores de saúde", originada das transcrições das falas durante as rodas de conversa, com o auxílio do software IRAMUTEQ (Interface de R pour les Analyses Multidimensionnelles de Textes et de Questionnaires), encontrou 9.193 ocorrências de palavras, sendo 1776 formas distintas com a frequência média de 5 palavras para cada forma, sendo este critério utilizado como ponto de corte para a inclusão no dendograma como mostra a figura 2.

Figura 2. Dendrograma gerado a partir das falas dos trabalhadores da saúde sobre questões ligadas aos portadores de Diabetes. Natal-RN. 2018.

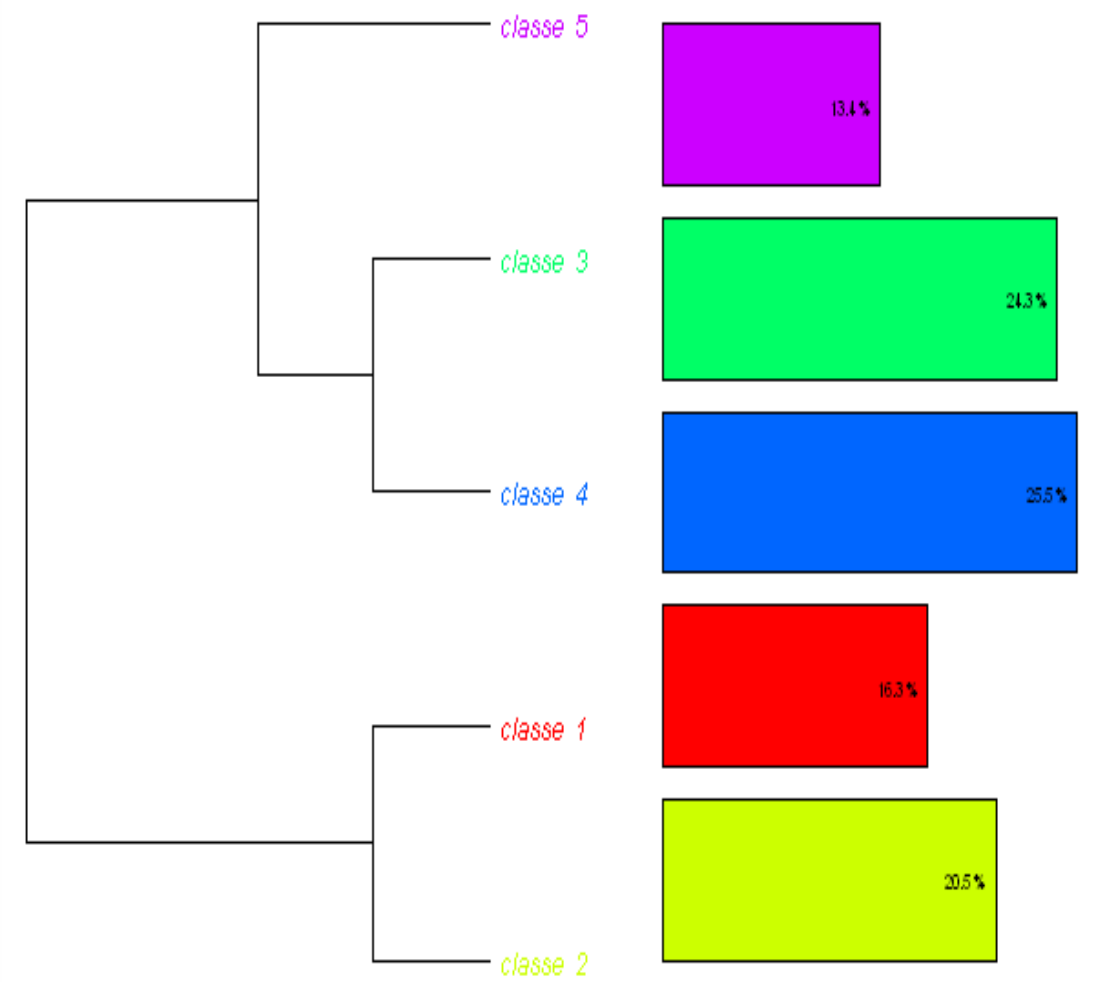

Esse corpus foi dividido em 260 unidades de contexto elementares (UCE) e destas 239, ou seja, 91,92\% do total de palavras foram equiparadas através de classificação hierárquica descendente (CHD) de segmentos de texto de tamanhos diferentes, indicando o grau de semelhança no vocabulário das cinco classes resultantes. Inicialmente, o corpus se dividiu primeiro formando a classe 5, depois houve uma divisão em classes 3 e 4 e, numa última partição, originou as classes 1 e 2 . 
Essa divisão de classes foi resultado dos agrupamentos de palavras com grau alto de semelhança.

A Classe 5 representou $13,4 \%$ do corpus e suas palavras mais representativas foram "gente", "coisa", "assim", "falar", "atividade" e "ficar". A palavra "gente", na realidade, utilizada no discurso diário de uma unidade de saúde, quase sempre não inclui realmente todas as pessoas, o popular e o científico. É uma representação plural voltada para o cotidiano do trabalhador no processo de trabalho em saúde, conforme as falas abaixo:

Por que a gente está ensinando a ter cuidado com os pés (Trabalhador 3).

A gente avalia os pés de todos (Trabalhador 5).

A gente gostaria de um esforço maior para vir nesse dia (Trabalhador 7).

Conversar sobre as coisas que melhoram a saúde da gente (Trabalhador 9).

A Classe 1 representou 16,3\% do corpus analisado e as palavras que mais contribuíram foram "ter", "ser", "dizer", "coisa", "grupo" e "pessoa". A obrigatoriedade e rigidez de palavras como "ter" e "ser" não nos fala muito sobre educação popular como as palavras "dizer", "pessoa" e "grupo". O educador deve ouvir mais as pessoas do grupo ao contrário de ficar determinando que se deve ser ou fazer, em relação a isso ou aquilo, fazendo continuamente uma prescrição de comportamento.

Por sua vez, a Classe 2 sinalizou 20,5\% de todo o corpus e as palavras mais destacadas foram "fazer", "estar", "grupo", "atividade", "dia" e "diabético". Convém enfatizar que a palavra "fazer" é a quinta mais citada em todo o processo de análise. Ninguém pergunta se o usuário quer e se não quiser, por quê? Esse tradicional modo pedagógico, autoritário, prescritivo e normativo de pensar saúde, deve ir sendo 
quebrado e desconstruído aos poucos, passando a dar maior espaço para Educação Popular em Saúde em nossas ações educativas4.

Já a Classe 3 representou $24,3 \%$ do total do corpus e as palavras que mais se repetiram foram: "ir", "querer", "tirar", "pé", "perna" e "levantar". É real e rotineiro o trabalhador de saúde insistir com o diabético para que cuide do pé e da perna. É um trabalho árduo no pensamento do orientador/prescritor. O querer, o desejar comer algo e não poder é muito forte e transforma os diabéticos em pessoas tristes e revoltadas. É nesse momento da vida do diabético que poderíamos trabalhar a pedagogia da condição humana e a liberdade de escolha de práticas alegres e afetivas, porém embasadas cientificamente e com domínio técnico a serviço da sociedade.

Finalmente, na Classe 4 , temos $25,3 \%$ de todo o corpus com palavras "atividade", "pra", "usar", "físico", "dizer" e "pé". Essa é a classe mais representativa do conteúdo das transcrições e que se somada a classe 3 mostra uma representação de $49,6 \%$, indo para $50 \%$. Ressaltamos aqui a certeza de os trabalhadores ainda estarem utilizando, na grande maioria, práticas pedagógicas tradicionais e uma educação bancária durante as atividades de educação nos grupos.

O software permitiu ainda uma análise de similitude visualizando a ligação entre os elementos. Esse tipo de análise se baseia em buscar indicações de conexão entre as palavras. Constatamos que as palavras maiores e em negrito demonstraram sua importância para essa ligação, esclarecendo que, quanto maiores as palavras, maior a sua frequência e contribuição para a formação da árvore de conexões. $\mathrm{Na}$ figura 3 percebemos quão rígida é a fala do educador de saúde e como as palavras "ter", "ser", "estar" e "ir", em negrito, aparecem com uma frequência bastante alta. Notamos também como a profissão atravessa a fala do profissional, em palavras como "mundo", “pessoas", “sopa”, “arroz", "aveia”, esquecendo uma relação de iguais. 


\section{ciênncia
pural}

Figura 3. Análise de Similitude a partir das falas dos trabalhadores da saúde sobre questões ligadas aos portadores de Diabetes. Natal-RN. 2018.

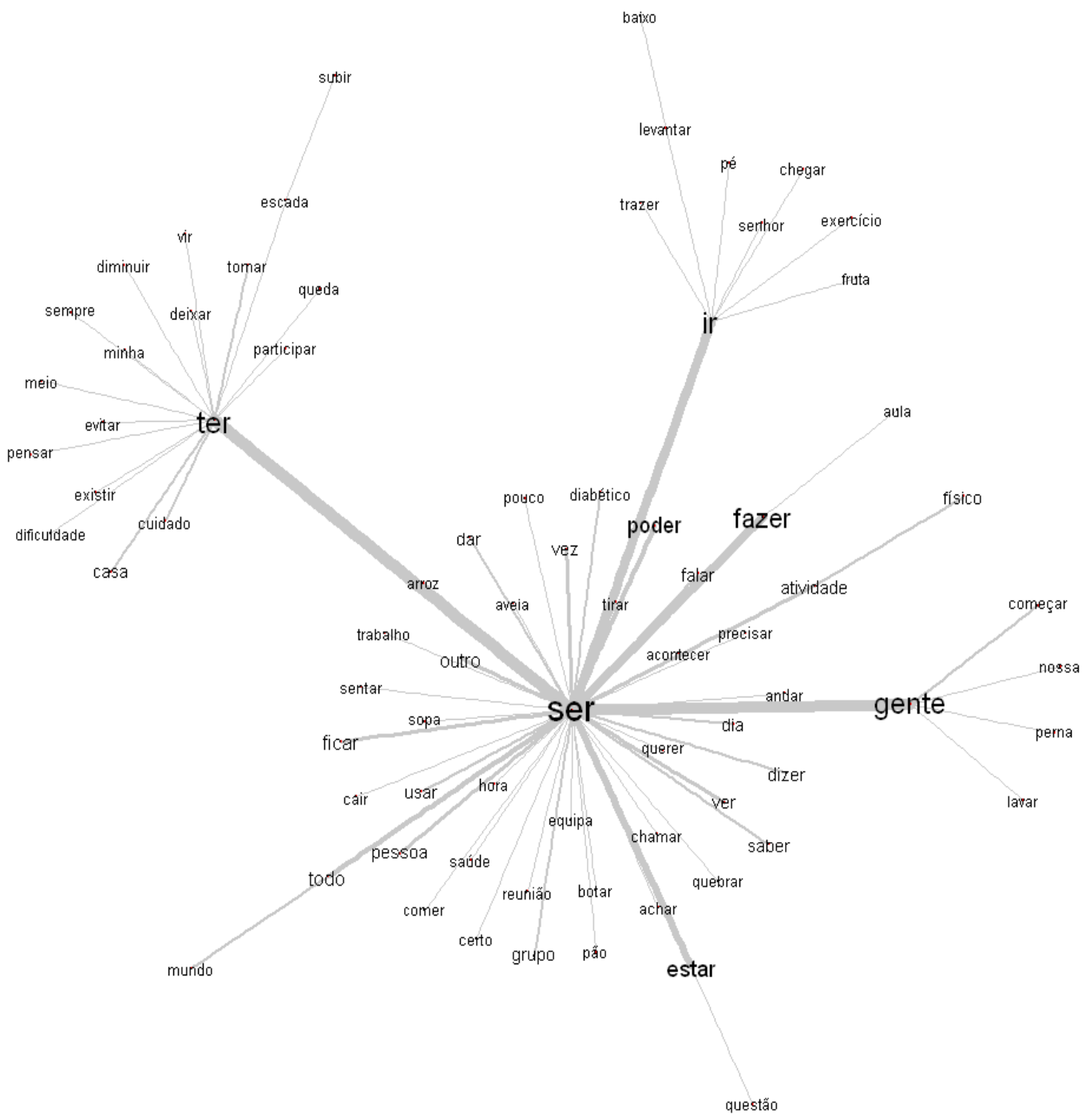

A ausência de palavras como "liberdade", "diálogo", "problematização", "humanização" e "reflexão crítica", não se destacando como elementos importantes na análise, revela uma lógica de educar muito distante das premissas da EPS nas ações educativas. A imposição de normas e comportamentos objetivando o modo de levar a 


\section{ciência
pural}

vida para alcançar mudanças pessoais é uma leitura errada da realidade, uma dimensão ultrapassada da Atenção Primária em Saúde .

Figura 4. Principais palavras da análise de similitude a partir das falas dos trabalhadores da saúde sobre questões ligadas aos portadores de Diabetes. NatalRN. 2018.

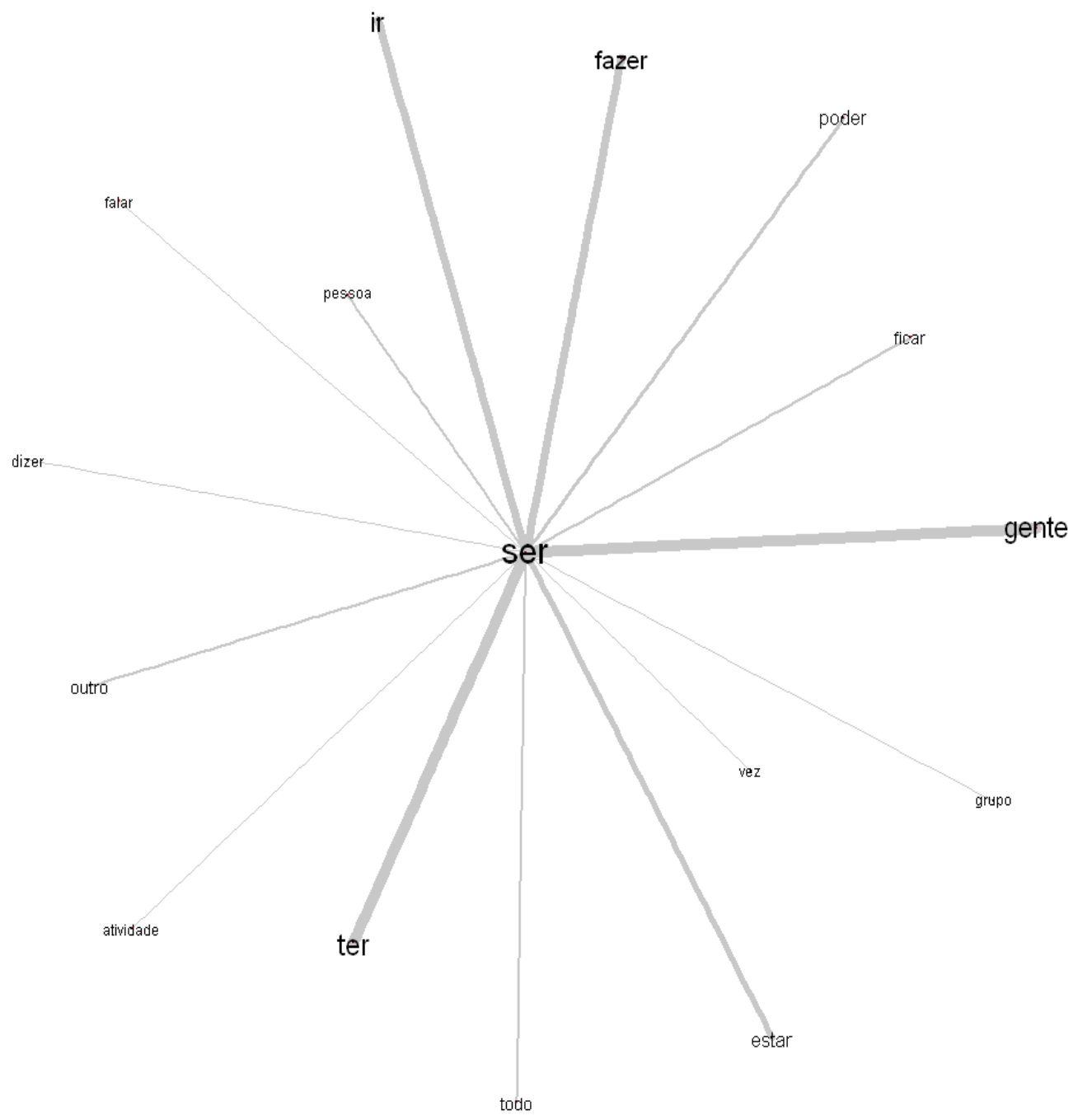


As palavras "ter" e "ser", conforme aparecem nas figuras 4 e 5, são bem características de trabalhadores de saúde e usadas sem muito critério na maioria das vezes, como se após uma frase apenas, toda a situação daquela pessoa se transformasse, sem sequer uma reflexão crítica. A nuvem de palavras é um recurso que agrupa e organiza graficamente as palavras-chave de um corpus em função da sua frequência, sendo assim, uma análise lexical mais simples, porém de visualização interessante. O leitor lê rapidamente as palavras-chave de um corpus.

Figura 5. Nuvem de Palavras gerada a partir das falas dos trabalhadores da saúde sobre questões ligadas aos portadores de Diabetes. Natal-RN. 2018.

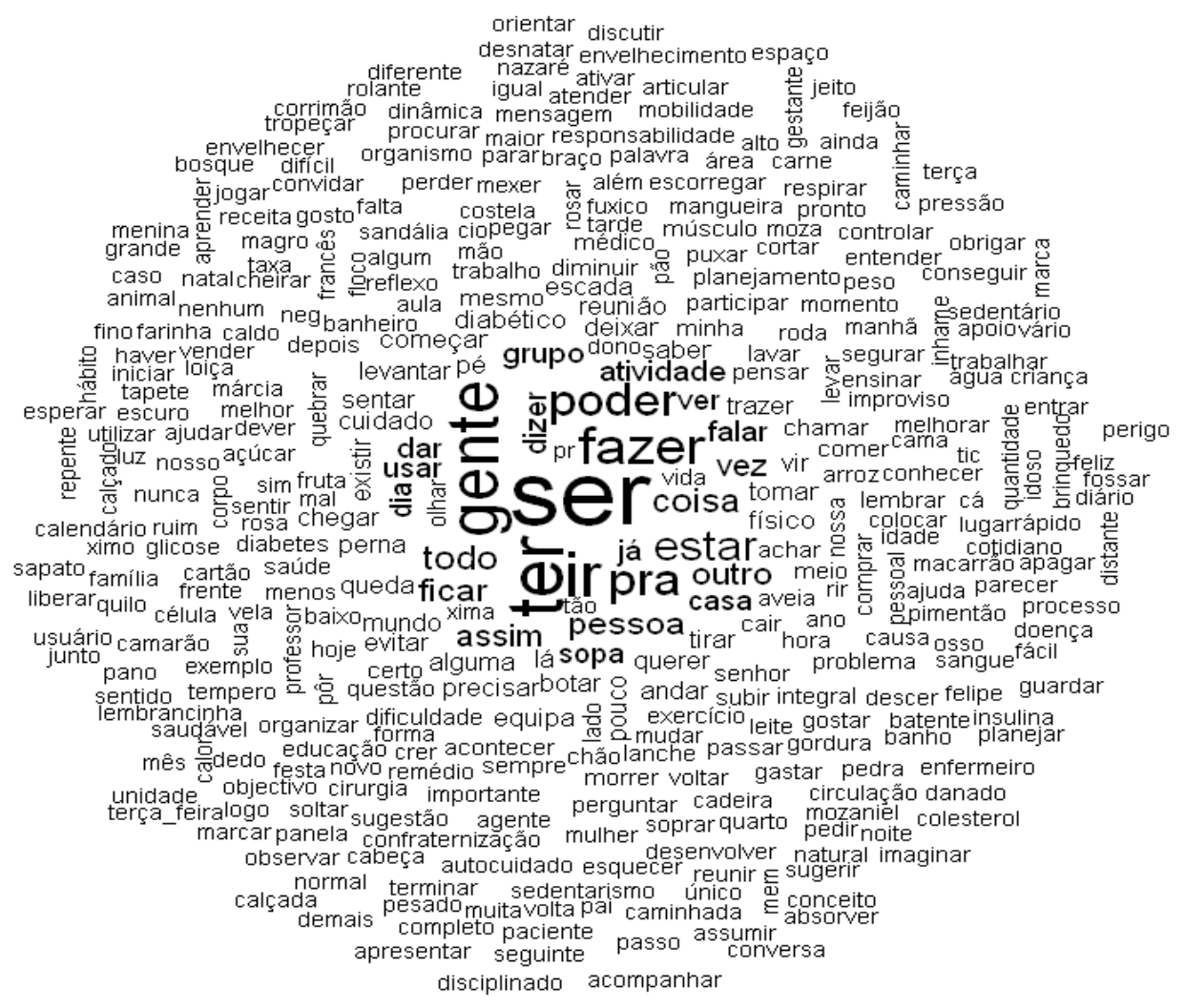


Os temas abordados nas rodas de conversa, durante os momentos de contextualização e de problematização, foram: o cuidado com o pé diabético; o significado de conviver com a diabetes; os sentidos da atividade física para o controle da diabete; alimentação saudável; prevenção de quedas; uso racional de medicamentos. As discussões sobre alimentação saudável foi um dos pontos mais relevantes para o diabético, seguido da própria doença e do estado de espírito para enfrentamento de suas dificuldades cotidianas. A atividade física e os medicamentos apareceram em menor destaque.

Enfatizamos que a dificuldade na adequação alimentar do diabético é a responsável por toda a diminuição da alegria de viver. A diabetes gera muito sofrimento psíquico no seu portador, passando despercebida pelos trabalhadores que, muitas vezes de maneira automatizada, atribui a não adesão à dieta como uma postura de fraqueza por parte do usuário, nos momentos de recaídas. Todo esse contexto leva a sofrimentos psíquicos que são trazidos à baila nos encontros em grupos, pela oportunidade de refletir, compartilhar experiências e saberes, com a finalidade de superar situações limitantes do seu viver ${ }^{25}$. Faz-se necessário que atividades educativas mais participativas sejam desenvolvidas nos grupos de educação em saúde levando-os a reflexão sobre a importância da mudança de comportamento e atitudes a fim de melhorar autoestima, vontade para aprender, controlar a patologia, proporcionando uma convivência mais feliz no meio familiar e social.

Quem tem essa doença não pode ter alegria, não pode ter emoção, preocupação, e eu tenho tudo isso. Eu tenho raiva, quase todos os dias. Eu já perdi até o jeito de viver (Usuário 12).

Não sou feliz sentindo-me com diabetes, mas é consequência da vida. Sinto muita saudade de doce (Usuário 1).

Diabetes me tira o prazer de me alimentar como gosto (Usuário 2).

A minha visão eu estou lutando para recuperar. A minha filha mais nova já está com diabetes, mas nunca é tarde para ser feliz (Usuário 9). 


\section{ciênncia
purál}

$\mathrm{Na}$ análise, surgiram a medicação e a atividade física como questões sem maior importância, apesar de terem sido citadas durante as rodas de conversa. Quando cruzamos essas informações com o perfil clínico dos diabéticos confirmamos a realização de pouca atividade física, o sedentarismo gerando um elevado grau de obesidade.

A solidariedade, o fortalecimento e o aumento do nível de consciência crítica sobre a realidade, podem tornar os grupos mais capazes para outros enfrentamentos, criando situações de lutas e ações participativas, para que a complexidade dos problemas relacionados à Diabetes Mellitus (DM) venha a ser enfrentados com mais naturalidade.

A educação em saúde visa à integração dos saberes, estabelecendo os conhecimentos e práticas de saúde que podem ser realizados para o fortalecimento de vínculos sociais. Considerando os saberes originados de sensações, emoções, concepções, narrações e sonhos, que junto com os aspectos técnico-científicos compõem a condição da natureza humana, notamos um aumento na desenvoltura dos usuários participantes, durante as rodas de conversa, influenciados pelo uso de metodologias mais participativas, gerando tímidas reflexões críticas sobre a realidade.

\section{Conclusões}

Essa pesquisa permitiu uma melhor compreensão da realidade dos grupos de diabéticos e dos trabalhadores de saúde durante o desenvolvimento de ações educativas através de metodologias participativas. Sugerimos a contínua capacitação dos trabalhadores para evitar a reprodução de ações baseadas apenas na boa vontade,

na intuição e no bom senso. $O$ processo educativo deve ser construído com planejamento adequado.

Percebemos que o encontro entre as culturas popular e científica, durante as le conversa, buscou a transformação das práticas pedagógicas tradicionais em 
práticas capazes de desenvolver o autocuidado mais responsável por parte dos participantes. As atividades educativas em grupo, orientadas pela participação, respeito e diálogo, entre os atores sociais, podem reorientar a lógica assistencialista dos serviços de saúde. No entanto, as reuniões realizadas pelos trabalhadores de saúde na sua maioria apenas cumprem uma normatização e ficaram aquém dos princípios de uma pedagogia libertadora.

Enfatizamos a importância de intervenções multiprofissionais, no contexto da ESF, para promover hábitos e estilos de vida saudáveis, prevenindo complicações do diabetes e proporcionando melhoria da qualidade de vida dos sujeitos. É fundamental o uso de metodologia ativas centrada nos participantes, a exemplo de jogos, dinâmicas e debates, com a finalidade, além do ensino e aprendizagem, de superar a sensação de isolamento social, nos espaços de encontro para expressão de sentimentos, fragilidades, limitações, infelicidades, tristezas e saudades, em busca de fomentar a fé e a força de vontade para seguir na luta cotidiana para controle da DM.

Faz-se necessário que atividades educativas mais participativas sejam desenvolvidas nos grupos de educação em saúde levando-os a reflexão sobre a importância da mudança de comportamento e atitudes a fim de melhorar autoestima, vontade para aprender, controlar a patologia, proporcionando uma convivência mais feliz no meio familiar e social. A metodologia do diálogo, da problematização e o reconhecimento das diversas formas de construir saberes são pertinentes para contribuir para o protagonismo dos sujeitos.

\section{ferências}

1. Faria HTG, Santos MA, Arrelias CCA, Rodrigues FFL, Gonela JT, Teixeira CRS et al Adesão ao tratamento em diabetes mellitus em unidades da Estratégia Saúde da Família. Rev. esc. enferm. USP. 2014 Apr; 48(2):257-263.

2. Rouquayrol MZ, Silva MGC. Epidemiologia \& saúde. 7ed. Rio de Janeiro: MedBook; 
3. Brasil. Estratégias para o cuidado da pessoa com doença crônica: diabetes mellitus. Brasília: Ministério da Saúde; 2013.

4. Vasconcelos EM, Cruz PJSC, Prado EV. A contribuição da Educação Popular para a formação profissional em saúde. Interface (Botucatu). 2016 Dec; 20(59):835-838.

5. Gusso G, Lopes JMC. Tratado de medicina de família e comunidade: princípios, formação e prática. Porto Alegre: Artmed; 2012.

6. Jahn AC, Guzzo PC, Costa MC, Silva EB, Guth EJ, Lima SBS. Educação popular em saúde: metodologia potencializadora das ações do enfermeiro. REUFSM. 2012; 2(3):547-52.

7. Souza KM, Goldschmidt IL, Bornstein VJ, Acioli S. Práticas pedagógicas de Educação Popular em Saúde e a formação técnica de Agentes Comunitários de Saúde no município do Rio de Janeiro, Brasil. Interface (Botucatu). 2014; 18(Suppl 2):15131522.

8. Flisch TMP, Alves RH, Almeida TAC, Torres HC, Schall VT, Reis DC. Como os profissionais da atenção primária percebem e desenvolvem a Educação Popular em Saúde?. Interface (Botucatu). 2014; 18(Suppl 2):1255-1268.

9. Sampaio J, Santos GC, Agostini M, Salvador AS. Limites e potencialidades das rodas de conversa no cuidado em saúde: uma experiência com jovens no sertão pernambucano. Interface (Botucatu). 2014; 18(Suppl 2):1299-1311.

10. Gomes LF, Campos VM, Sandoval RA. Prevalência de amputação em decorrência de complicações do pé diabético. Rev. Digital, Buenos Aires. 2008; 13(124).

11. Sociedade Brasileira de Diabetes (SBD). Algoritmo para o tratamento do diabetes tipo 2 - atualização. Posicionamento oficial SBD. 2018;(2).

12. Carlesso GP, Gonçalves MHB, Moreschi JD. Avaliação do conhecimento de pacientes diabéticos sobre medidas preventivas do pé diabético em Maringá (PR). J. vasc. bras. 2017 June; 16(2):113-118.

13. Poletti NAA. O cuidado de enfermagem a pacientes com feridas crônicas: a busca de evidências para a prática. [dissertação]. Ribeirão Preto (SP): Escola de Enfermagem de Ribeirão Preto/USP; 2000.

14. Freire P. Educação como prática da liberdade. 14. Ed. Rio de Janeiro: Paz e Terra; 
15. Vieira AJH, Batalloso JM, Moraes MC. A Esperança da Pedagogia Paulo Freire: Consciência e Compromisso. Rio de Janeiro: Liber Livro; 2012.

16. Gomes LB, Merhy EE. Compreendendo a educação popular em saúde: um estudo na literatura brasileira. Cad. Saúde Pública. 2011 Jan; 27(1):7-18.

17. Stotz EM, David HSL, Wong Un JA. Educação popular e saúde: trajetória, expressões e desafios de um movimento social. Revista APS. 2005; 8(1):49-60.

18, Thiollent MJM. Metodologia da pesquisa-ação. 17ed. São Paulo: Editora Cortez; 2009.

19. Freire P. Pedagogia da autonomia. 37ed. Rio de Janeiro: Paz e Terra; 2008.

20. Nóbrega SM, Coutinho MPL. O teste de associação livre de palavras. In: Coutinho MPL, Oliveira FB, Fortunato ML. Representações Sociais: abordagem interdisciplinar. João Pessoa: Editora Universitária/UFPB; 2003. p.67-77.

21. Bardin JL. Análise de Conteúdo. São Paulo: Edições 70; 2011.

22. Ratinaud, P, Marchand P. Application de la méthode ALCESTE à de "gros" corpus et stabilité des "mondes lexicaux": analyse du "Cable-Gate" avec IraMuTeQ. In: Actes des 11eme Journées internationales d'Analyse statistique des Données Textuelles. 2012; p. 835-844. Liège, Belgique. Retrieved April 15, 2018.

23. Falkenberg MB, Mendes TPL, Moraes EP, Souza EM. Educação em saúde e educação na saúde: conceitos e implicações para a saúde coletiva. Ciênc. saúde coletiva. 2014; 19(3):847-852.

24. Torres HC, Pereira FRL, Alexandre LR. Evaluation of the educational practices in promoting self-management in type 2 diabetes mellitus. Rev. esc. enferm. USP. 2011; 45(5):1077-1082.

25. Bernini LS, Barrile SR, Mangili AF, Arca EA, Correr R, Ximenes MA, et al. O impacto do diabetes mellitus na qualidade de vida de pacientes da Unidade Básica de Saúde. Cad. Bras. Ter. Ocup., São Carlos. 2017; 25(3):533-541.

Submetido: $25 / 03 / 2019$

Aceito: 25/08/2019 